\title{
ASPECTOS ETNOBOTÂNICOS DA FITOTERAPIA POPULAR NA COMUNIDADE QUILOMBOLA CONCEIÇÃO DE MIRINDEUA, MOJU-PA
}

\author{
Jaqueline dos Santos Nascimento ${ }^{1}$, Isabela de Oliveira Carvalho ${ }^{1}$, Mariana Casari Parreira ${ }^{1}$, Sebastião Ribeiro
} Xavier Junior ${ }^{2}$, Daniely Alves Almada ${ }^{3}$, Wellington Moraes Ferreira ${ }^{1}$, Francisco Tiago de Vasconcelos Melo ${ }^{1}$

\begin{abstract}
RESUMO - A Amazônia possui uma vasta riqueza de recursos naturais, dentre estes se encontram as espécies de plantas medicinais que tem um grande valor cultural presente para muitos povos tradicionais. No entanto, ainda existem várias espécies desconhecidas ou pouco elucidadas pela ciência que são bastante utilizadas por populações tradicionais. Diante disso, o presente estudo objetivou fazer um levantamento etnobotânico de plantas medicinais utilizadas pelos moradores da comunidade quilombola de Conceição de Mirindeua utilizando entrevistas semi-estruturadas. As entrevistas aconteceram no período de outubro a novembro de 2019, com 25 (vinte e cinco) moradores com idades entre 28 a 77 anos. Os resultados obtidos mostram que a maioria dos entrevistados ( $64 \%$ do total) possuem apenas o ensino fundamental incompleto e renda familiar de até 1 salário mínimo. Foram citadas 86 etnoespécies, ocorrendo a identificação de 83 pelo laboratório de Botânica da Embrapa/Belém, sendo 1 a nível de família, 68 a nível de família e espécie e somente 14 a nível de família e gênero, tendo destaque para a família Lamiaceae com 16 espécies. A forma de preparo mais citada foi o chá e as folhas tiveram maior representatividade como parte utilizada.
\end{abstract}

Palavras chave: plantas medicinais, populações tradicionais, toxicidade.

\section{ETHNOBOTANICAL ASPECTS OF PHYTOTHERAPY POPULAR IN THE QUILOMBOLA COMMUNITY CONCEIÇÃO DE MIRINDEUA, MOJU-PA}

\begin{abstract}
The Amazon possesses a vast wealth of natural resources, among which are the species of medicinal plants that have a cultural value well present for many traditional peoples. However, there are still several species unknown or little elucidated by science that are widely used by traditional populations. In view of this, the present study aimed to make an ethnobotanical survey of medicinal plants used by residents of the quilombola community of Conceição de Mirindeua using semi-structured interviews. The interviews took place from October to November 2019, with 25 (twenty-five) residents between the ages of 28 and 77 . The results obtained show that the majority of interviewees ( $64 \%$ of the total) have only incomplete primary education and family income of up to 1 minimum wage. Eighty-six ethnospecies were cited, and 83 were identified by the Embrapa/Belém Botany Laboratory, 1 at the family level, 68 at the family and species level, and only 14 at the family and genus level, with emphasis on the Lamiaceae family with 16 species. The most cited form of preparation was the tea and the leaves had greater representation as part used.
\end{abstract}

Keywords: medicinal plants, toxicity, traditional populations.

\footnotetext{
${ }^{1}$ Universidade Federal do Pará; E-mail: jaqueline.agronomia@hotmail.com

${ }^{2}$ EMBRAPA Amazônia Oriental.

${ }^{3}$ Universidade Nove de Julho.
} 


\section{INTRODUÇÃO}

A fitoterapia popular está relacionada à utilização de plantas medicinais, que fazem parte da cultura de uso da medicina popular, constituindo uma junção de saberes ligados a diversos grupos sociais que repassam esses conhecimentos de geração a geração, fazendo parte da história da humanidade e exercendo papel importante na construção do conhecimento científico e tradicional, uma vez que o relacionamento do ser humano com as plantas, numa perspectiva etnobotânica, é relatado desde a antiguidade até os tempos atuais, sendo de suma importância no atendimento primário a saúde, principalmente para populações em vulnerabilidade social (Bruninget et al., 2012; Cassas et al., 2016).

Dessa forma, pode-se constatar que o conhecimento sobre esses fitoterápicos se mostra de grande valia e utilidade tanto para o mundo científico como para a sociedade de um modo geral, inclusive, para as populações tradicionais, que mantém intrínseca ligação com a natureza e forte dependência dos recursos naturais tanto para a subsistência familiar como também para a reprodução sociocultural desses povos. Para estes, a utilização de plantas medicinais configura-se, na maioria das vezes, como a única alternativa para prevenir ou tratar doenças, através do seu poder curativo, em razão de tais comunidades geralmente estarem localizadas distantes dos centros urbanos (Giraldi \& Hanazaki, 2010).

Segundo Sales et al. (2015), o Brasil ocupa a primeira posição no ranking em matéria de diversidade vegetal do planeta e amplo sócio biodiversidade, com enorme potencial no desenvolvimento da fitoterapia. Os autores também enfatizam que a utilização de plantas medicinais é decorrente de um histórico processo de produção e reprodução de diversos saberes e práticas de comunidades tradicionais, entre elas os quilombos, que desempenham um importante papel na preservação das tradições etnobotânicas africanas no continente americano (Oliveira et al., 2015).

Neste caminho, os povos da Amazônia possuem vasto conhecimento sobre o uso e manejo das plantas nos quintais (Coelho-Ferreira \& Jardim, 2005). Para Winklerprins \& Oliveira (2010) esses espaços envolvem um intercâmbio de material genético que atrela a diversidade existente nos ecossistemas naturais às culturas ou tradições das comunidades humanas locais. Os quintais amazônicos são unidades produtivas manejadas durante anos que fornecem meios de subsistência às comunidades rurais e urbanas (Borba \& Macedo, 2006).
Em razão disso, este trabalho teve como objetivos fazer o levantamento, mediante identificação e análise, das espécies vegetais utilizadas para fins medicinais, assim como compreender os principais aspectos inerentes à prática da fitoterapia popular na comunidade quilombola Conceição de Mirindeua.

\section{MATERIAL E MÉTODOS}

Área de estudo

A pesquisa foi desenvolvida na comunidade quilombola Conceição de Mirindeua, pertencente ao território do Jambuaçu, ás margens da rodovia dos quilombolas PA 252, a 32 (trinta e dois) $\mathrm{Km}$ da sede do município de Moju-PA (Figura 1).

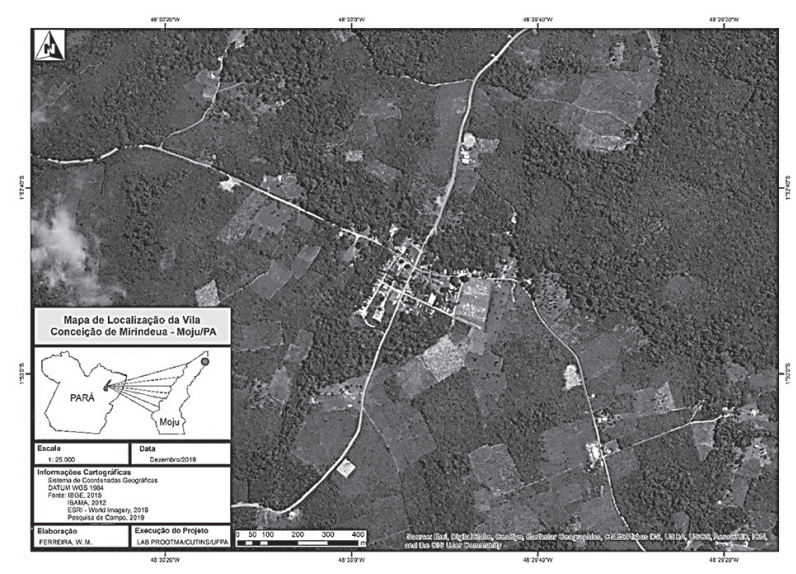

Figura 1 - Localização da comunidade quilombola Conceição de Mirindeua, Moju-PA.

Fonte: Ferreira (2019).

Este município possui população estimada em 70.018 (setenta mil e dezoito) habitantes distribuídos em 7,70hab $/ \mathrm{km}^{2}$ de extensão territorial (IBGE, 2010). É localizado no Nordeste do Estado do Pará, possuindo uma área de 9.094,10 km2. Possui em suas coordenadas geográficas aproximadamente $02^{\circ} 29^{\prime} 44.5^{\prime \prime} \mathrm{S}$ e 048 48'05.8'W. De acordo com a classificação de Köppen, o clima do município é quente e úmido, com temperatura média anual na faixa de $25^{\circ} \mathrm{C}$. A umidade relativa do ar se aproxima de $85 \%$ e, sendo de janeiro a junho a sua maior época de pluviosidade (Pimenta, 2018).

A comunidade foi fundada por volta de 1860 , segundo relatos dos moradores mais antigos. Esta tem titulação de domínio coletivo, segundo o Instituto de 
Terras do Pará -ITERPA. O principal documento de reconhecimento dessa comunidade como remanescente de quilombo é a Certidão Palmares.

\section{Coleta de dados}

Para o levantamento de dados foi utilizado um questionário semiestruturado com perguntas relacionadas a situação socioeconômica e sociodemográfica dos moradosres, além de informações acerca da utilização de plantas medicinais.

A pesquisa foi realizada durante os meses de outubro e novembro de 2019, utilizando-se a técnica de amostragem proposta por Bailey (1994), cuja metodologia é chamada "bola de neve" (snowball), e baseia-se na escolha do primeiro especialista entrevistado. Este, por sua vez, indica o próximo, e assim por diante, até chegar ao final da pesquisa, onde todos os especialistas indicados sejam entrevistados.

A coleta das espécies dos vegetais se deu logo após a entrevista, objetivando conseguir as melhores amostras com o intuito de se obter o melhor resultado possível de identificação. Em seguida, as amostras foram colocadas em jornal para preparo das exsicatas. Após essas etapas, as amostras foram enviadas para análise no laboratório de botânica da EMBRAPA Amazônia Oriental.

Os dados levantados foram compostos por nome popular, parte utilizada, forma de uso e finalidade de uso. Para a tabulação dos dados, utilizou-se o Programa Excel 2010 .

\section{RESULTADOS E DISCUSSÃO}

Foram entrevistados 25 (vinte e cinco) moradores com faixa etária variando de 28 a 77 anos, com média de idade de 58 anos. Entre os entrevistados $80 \%$, são do sexo feminino enquanto que $20 \%$ são do sexo masculino. Vásquez et al. (2014), em trabalho sobre etnobotânica de plantas medicinais realizadas em comunidades ribeirinhas no Amazonas, relataram que dos 164 moradores entrevistados durante as pesquisas, 102 foram mulheres e 62 homens, externando a importância destas no uso e transmissão de saberes sobre plantas medicinais.

Em relação a atividade laboral, $92 \%$ se auto definem como agricultores, $4 \%$ como funcionários públicos e $4 \%$ pertencem ao setor privado. Quanto à escolaridade dos entrevistados, observou-se que a maioria, $64 \%$ possui apenas o ensino fundamental incompleto e $20 \%$ são analfabetos. Em relação aos demais níveis de escolaridade,
$4 \%$ possui o ensino superior completo, $4 \%$ possui o ensino médio incompleto, $4 \%$ possui o ensino médio completo e $4 \%$ possui curso técnico.

De acordo com os relatos de alguns entrevistados, o nível baixo de escolaridade na comunidade está diretamente ligado as dificuldades de acesso ao ensino nas zonas rurais onde, há alguns anos, existiam, no máximo, escolas de nível fundamental. Segundo eles, nem todos tinham condições de se deslocar para o município mais próximo ou para a capital para ter acesso ao ensino. Tal realidade é evidente também em duas comunidades quilombolas de Goiás, onde houve o predomínio da baixa escolaridade entre os entrevistados, com a maior parte da população possuindo somente o ensino fundamental incompleto $(51,2 \%$ e $58,5 \%$, respectivamente) (Santos \& Silva, 2010). Esta situação é oriunda, principalmente, da falta de escolas nesses territórios, além da dificuldade de acesso e permanência em pólos ou cidades vizinhas.

Segundo dados da Secretaria de Políticas de Promoção da Igualdade Racial - SEPPIR, do Governo Federal, cerca de $23,5 \%$ dos quilombolas do país não sabem ler, refletindo ainda hoje o processo histórico excludente do qual esses indivíduos fazem parte, sem acesso, comumente, a direitos básicos que asseguram a dignidade humana, resultando na falta de condições para permanência em estabelecimentos de ensino ou mesmo a falta ou precariedade destes no seu território (SEPPIR, 2012).

Em relação à renda mensal dos entrevistados, $48 \%$ afirmou que recebem 1 salário mínimo, 40\% informaram ter renda menor que 1 salário mínimo, e 12\% afirmaram possuir renda maior que 1 salário mínimo.

Esses resultados foram inferiores aos obtidos por Giraldi \& Hanazaki (2010) em seu trabalho sobre o uso e conhecimento tradicional de plantas medicinais no Sertão do Ribeirão, Florianópolis-SC, onde a maioria dos entrevistados sobrevive com uma renda familiar que varia de 2 a 3 salários mínimos (62\%) e foram semelhantes aos encontrados por Santos \& Silva (2014) onde constataram que a maioria dos entrevistados $(57,9 \%$ e $57,1 \%$, respectivamente) possuem renda em torno de 1 a 2 salários mínimos.

A renda familiar baixa está associada direta ou indiretamente ao nível de escolaridade dos indivíduos, pois esta pode influenciar o tipo de ocupação destes e, consequentemente a renda familiar dos mesmos. Esta percepção também foi descrita por Sá (2010) e Santos \& Silva (2014). Estes afirmam que a renda familiar é uma variável de suma importância para avaliar as condições de 
vida de determinada população, pois é o fator limitante do acesso a diversos bens de consumo, inclusive de acesso a serviços de saúde, e está relacionada ao tipo de ocupação familiar. Além disso, dados da Secretaria de Políticas de Promoção da Igualdade Racial - SEPPIR (2012), revelam que grande parte das famílias quilombolas do país, cerca de $75,6 \%$ do total, estão em situação de vulnerabilidade socioeconômica ou de extrema pobreza.
Foram citadas pelos entrevistados 86 Etnoespécies, das quais 83 foram identificadas, sendo 1 a nível de família, 68 a nível de família e espécie e somente 14 a nível de família e gênero, totalizando o número de 41 famílias, tendo destaque para a família Lamiaceae com 16 espécies, Anacardiaceae com cinco espécies, Asteraceae, Zingiberacea e Bignoniaceae ambas com quatro espécies (Tabela 1).

Tabela 1 - Plantas utilizadas para fins medicinais na comunidade quilombola de Conceição do Mirindeua, Moju-PA

\begin{tabular}{|c|c|c|c|c|}
\hline FamílialNome científico & Nome popular & Parte utilizada & Forma de preparo & Finalidade de uso \\
\hline \multicolumn{5}{|l|}{ LAMIACEAE } \\
\hline Mentha Spicata L. & Hortelãzinho & Folha & Chá & \\
\hline Pogostemom heyneanus Benth. & Oriza & Folha & Chá/banho & Banho cheiroso \\
\hline Mentha sp. & Cibalena & Folha & Chá & Febre \\
\hline Occimum gratissimum $\mathrm{L}$. & Favacão & Folha & Banho & Gripe \\
\hline $\begin{array}{l}\text { Scutellaria agrestis A.St.-Hil. ex } \\
\text { Benth. }\end{array}$ & verga morta & Folha & Chá & Dores em geral \\
\hline $\begin{array}{l}\text { Aeollanthus suaveolens Mart. ex } \\
\text { Spreng }\end{array}$ & Catinga de mulata & Folha & Chá & AVC \\
\hline Plectranthus sp. & Pirarucu & Folha & Chá/xarope & Asma \\
\hline Plectranthus L'Hér. & Boldo & Folha & Chá & Dor estomacal \\
\hline Scutellaria L. & Hortelã roxo & Folha & Chá & Dor estomacal \\
\hline Mentha sp. & Hortelã grande & Folha & Chá/xarope & Tosse \\
\hline Plectranthus barbatus Andr. & Anador & Folha & Chá & Dores em geral \\
\hline Mentha arvensis $\mathrm{L}$. & Hortelã pimenta & Folha & Chá & Gases de criança \\
\hline $\begin{array}{l}\text { Plectranthus amboinicus (Lour.) } \\
\text { Spreng. }\end{array}$ & Hortelã do maranhão & Folha & Chá & Dores em geral \\
\hline $\begin{array}{l}\text { Aeollanthus suaveolens Mart. } \\
\text { exSpreng. }\end{array}$ & Caatinga da mulata & Folha & Chá & AVC \\
\hline Ocimum minimum $\mathrm{L}$. & Mangericão & Folha & Chá/banho & Gripe \\
\hline Stemon linearis Pogo (Benth.) Kuntze & Oriza & Folha & Chá/banho & Banho cheiroso \\
\hline \multicolumn{5}{|l|}{ COSTACEAE } \\
\hline Costus spicatus (Jacq.) Sw. & Canarana & Folha/planta & Chá & Infecção urinária \\
\hline \multicolumn{5}{|l|}{ PIPERACEAE } \\
\hline Piper divaricatum G.Mey. & Pau de angola & Folha & Chá/banho & Constipação \\
\hline Piper sp. & Óleo elétrico & Folha & Chá/banho & Dores em geral \\
\hline Piper umbellata (L.) Miq. & Calipe & Folha & Chá & Febre \\
\hline \multicolumn{5}{|l|}{ MYRTACEAE } \\
\hline Eucalyptus sp. & Eucalipto & Folha & Chá & Febre \\
\hline Psidium guajava $\mathrm{L}$. & Goiaba & Folha & Chá & Diarreia \\
\hline Psidium guianense Pers. & Aracá & Folha & Chá & Diarréia \\
\hline
\end{tabular}


Tabela 1 - Cont.

\begin{tabular}{|c|c|c|c|c|}
\hline Família\Nome científico & Nome popular & Parte utilizada & Forma de preparo & Finalidade de uso \\
\hline \multicolumn{5}{|l|}{ PHYTOLACCACEAE } \\
\hline Petiveria alliacea $\mathrm{L}$. & Mucuracaá & Folha & Chá & Dores em geral \\
\hline \multicolumn{5}{|l|}{ ASTERACEAE } \\
\hline $\begin{array}{l}\text { Gymnanthemum amygdalinum } \\
\text { (Delile) Sch.Bip. ex Walp. }\end{array}$ & Boldo & Folha & Chá & Dor estomacal \\
\hline Vernonia scabra Pers. & Pau de muquim & Folha & Chá & Sarampo/tosse \\
\hline Mikania lindleyana DC. & Sucuriju & Folha & Chá & Dor estomacal \\
\hline Tagetes erecta $\mathrm{L}$. & Cravo & Folha & Sumo & AVC \\
\hline \multicolumn{5}{|l|}{ ACANTHACEAE } \\
\hline Justicia secunda Vahl & Forsangue & Folha & Chá & Anemia \\
\hline Justicia pectoralis Jacq. & Trevo & Folha & Chá/banho & Irritabilidade de criança \\
\hline \multicolumn{5}{|l|}{ PHYLLANTHACEAE } \\
\hline Phyllanthus caroliniensis Walter & Quebra pedra & Raiz & Chá & Pedra na vesícula \\
\hline \multicolumn{5}{|l|}{ RUTACEAE } \\
\hline Citrus aurantium $\mathrm{L}$. & Laranja da terra & Folha & Chá/banho & Constipação \\
\hline Ruta graveolens $\mathrm{L}$. & Arruda & Folha & Chá & Dor de cabeça \\
\hline Citrus limon L. & Limãozinho & Folha & Chá & Febre \\
\hline \multicolumn{5}{|l|}{ RUBIACEAE } \\
\hline Morinda citrifolia $\mathrm{L}$. & Noni & Fruta & Chá & Colesterol/emagrecimento \\
\hline Uncaria Schreb. & Unha de gato & Casca & Chá & Gastrite \\
\hline \multicolumn{5}{|l|}{ MORACEAE } \\
\hline Dorstenia asaroides Gardner & Apií & Raiz & Chá & Tosse/dor de garganta \\
\hline \multicolumn{5}{|l|}{ CRASSULACEAE } \\
\hline Kalancho pinnata (Lam.) Pers. & Pirarucu & Folha & Sumo & Dor estomacal \\
\hline \multicolumn{5}{|l|}{ ZINGIBERACEA } \\
\hline Zingiber officinale Roscoe & Gengibre & Raiz & Chá & Tosse \\
\hline Curcuma longa $\mathrm{L}$. & Mangarataia & Raiz & Chá/pó & Inchaços \\
\hline Alpinia $\mathrm{L}$. & Vindicar & Folha & Chá/banho & Inchaços \\
\hline Alpinia $\mathrm{L}$ & Croto & Folha & Chá & Hemorroidas \\
\hline \multicolumn{5}{|l|}{ IRIDACEAE } \\
\hline Eleutherine plicata (SW) Herb. & Marupazinho & Raiz & Chá & Hemorroidas \\
\hline \multicolumn{5}{|l|}{ ANACARDIACEAE } \\
\hline Mangifera indica L. & Mangueira & Casca & Chá & Diarreia \\
\hline Spondias mombin $\mathrm{L}$. & Taperebá & Nó & Pó & Erisipela \\
\hline $\begin{array}{l}\text { Anacardium giganteum Loudon ex } \\
\text { Steudel }\end{array}$ & Caju do mato & Casca & Chá & Úlcera \\
\hline Schinus sp. & Afasta espirito & Folha & Chá/banho & Mau olhado \\
\hline Anacardium occidentale L. & Caju & Flor & Chá & Diarreia \\
\hline
\end{tabular}


Tabela 1 - Cont.

\begin{tabular}{|c|c|c|c|c|}
\hline Família\Nome científico & Nome popular & Parte utilizada & Forma de preparo & Finalidade de uso \\
\hline \multicolumn{5}{|l|}{ BORAGINACEAE } \\
\hline $\begin{array}{l}\text { Varroniamulti spicata (Cham.) } \\
\text { Borhidi }\end{array}$ & Parica do Mato & Folha & Chá & Erisipela \\
\hline \multicolumn{5}{|l|}{ MALPIGHIACEAE } \\
\hline \multirow[t]{2}{*}{$\begin{array}{l}\text { Banisteriopsis caapi (Spruce ex } \\
\text { Griseb.) Morton }\end{array}$} & Cabi & Folha & Sumo & Alergia \\
\hline & General & Folha & Chá & Problema de coração \\
\hline \multicolumn{5}{|l|}{ BIGNONIACEAE } \\
\hline Tanaecium sp. & Curimbó & Cipó & Chá/banho & Banho \\
\hline Mansoa alliacea (Lam.)A.H.Gentry & Cipó alho & Folha/chá & Banho & Mau olhado \\
\hline $\begin{array}{l}\text { Fridericia chica (Bonpl.) } \\
\text { L.G.Lohmann }\end{array}$ & Pariri & Folha & Chá & Anemia \\
\hline $\begin{array}{l}\text { Handroanthus serratifolius (Vahl) } \\
\text { S.Grose }\end{array}$ & Pau d'arco & Casca & Chá & Gastrite \\
\hline \multicolumn{5}{|l|}{ MALVACEAE } \\
\hline Hibiscus rosa sinensislinn. & Pampolha & Flor & Chá/xarope & Tosse \\
\hline Hibiscus sabdariffa L. & Vinagreira & Folha & Sumo & $\mathrm{AVC}$ \\
\hline Gossypium arboreum L. & Algodão & Folha & Sumo & Asma \\
\hline \multicolumn{5}{|l|}{ EUPHORBIACEAE } \\
\hline Euphorbia tithymaloides L. & Coramina & Folha & Chá & Dor estomacal \\
\hline \multicolumn{5}{|l|}{ VITACEAE } \\
\hline Cissus sicyaides $\mathrm{L}$ & Pucá & Folha & Chá & AVC \\
\hline \multicolumn{5}{|l|}{ PLANTAGINACEAE } \\
\hline Plantago major $\mathrm{L}$. & Transagem & Folha & Chá & Dores em geral \\
\hline \multicolumn{5}{|l|}{ ARACEAE } \\
\hline Caladium bicolor (Aiton) Vent. & Brasileirinha & Folha & Chá & Asma \\
\hline Caladium bicolor (Aiton) Vent. & Trajá de sol & Raiz & Pó & Bicheira \\
\hline \multicolumn{5}{|l|}{ SIMAROUBACEAE } \\
\hline Quassia amara $\mathrm{L}$. & Quina & Folha & Chá & Frialdade \\
\hline \multicolumn{5}{|l|}{ POACEAE } \\
\hline Saccharum sp. & Cana-roxa & Folha & Chá/banho & Alergia \\
\hline \multicolumn{5}{|l|}{ VERBENACEAE } \\
\hline $\begin{array}{l}\text { Lippia alba (Mill.) N.E.Br. ex } \\
\text { P.Wilson }\end{array}$ & Carmelitano & Folha & Chá & Febre/lavagem intestinal \\
\hline \multicolumn{5}{|l|}{ CAPRIFOLIACEAE } \\
\hline Sambucus autralis Cham. \& Schltdl. & Sabugueiro & Folha & Chá & Sarampo \\
\hline \multicolumn{5}{|l|}{ ARISTOLOCHIACEAE } \\
\hline Aristolochia $\mathrm{L}$. & Sangue de cristo & Folha & Chá & Mau olhado \\
\hline
\end{tabular}


Tabela 1 - Cont.

\begin{tabular}{|c|c|c|c|c|}
\hline Família\Nome científico & Nome popular & Parte utilizada & Forma de preparo & Finalidade de uso \\
\hline \multicolumn{5}{|l|}{ FABACEAE } \\
\hline Dalbergia monetaria L.f. & Veronica & Casca & Chá & Infecção uterina \\
\hline \multicolumn{5}{|l|}{ PLANTAGINACEAE } \\
\hline $\begin{array}{l}\text { Canibia scoparioides (cham \& } \\
\text { scheltdl.) kuntze }\end{array}$ & Pataqueira & Folha & Chá & Dores em geral \\
\hline \multicolumn{5}{|l|}{ MELIACEAE } \\
\hline Carapa guianensis Aubl. & Andiroba & Fruto & Óleo & Antiflamatório \\
\hline \multicolumn{5}{|l|}{ ANNONACEAE } \\
\hline Annona muricata L. & Graviola & Folha & Chá & Câncer \\
\hline \multicolumn{5}{|l|}{ LYTHRACEAE } \\
\hline Lawsonia inermis L. & Mortinha & Folha & Chá & Vomito/diarreia \\
\hline \multicolumn{5}{|l|}{ ARECACEAE } \\
\hline Euterpe oleracea Mart. & Açaí & Raiz & Chá & Diarreia \\
\hline \multicolumn{5}{|l|}{ AMARANTHACEAE } \\
\hline $\begin{array}{l}\text { Dhysphania ambrosioides (L) } \\
\text { Mosyakim \& clemants }\end{array}$ & Mastruz & Folha & Sumo & Gastrite \\
\hline Alternanthera Forssk. & Miracelina de planta & Folha & Chá & Anemia \\
\hline Saccharum sp. & Corretne roxa & Folha & Chá & Hemorróidas \\
\hline \multicolumn{5}{|l|}{ PASSIFLORACEAE } \\
\hline Passiflora edulis Sims & Maracujá & Flor & Chá & Problemas de coração \\
\hline \multicolumn{5}{|l|}{ APIACEAE } \\
\hline Eryngium foetidum L. & Chicória & Folha & Chá/banho & Quebranto \\
\hline \multicolumn{5}{|l|}{ HYPERICACEA } \\
\hline Vismia guianensis (Aubl.) Choisy & Lacre & Folha & Chá & Colesterol \\
\hline \multicolumn{5}{|l|}{ MELASTOMATACEAE } \\
\hline Clidemia hirta & Catininga & Folha & Sumo & Alergia de criança \\
\hline \multicolumn{5}{|l|}{ SOLANACEAE } \\
\hline Capsicum frutescens $\mathrm{L}$. & Pimenta malagueta & Folha & Emplasto & Nascida \\
\hline \multicolumn{5}{|l|}{ AMARYLLIDACEAE } \\
\hline $\begin{array}{l}\text { Hippeastrum puniceum( Lam ) } \\
\text { kurtze }\end{array}$ & Sussena & Raiz & Xarope & Asma \\
\hline \multicolumn{5}{|l|}{ NÃO IDENTIFICADAS } \\
\hline & Força de sangue & Folha & Chá & Anemia \\
\hline & Copaiba & fruta & Óleo & Antiflamatório \\
\hline & Cipó catinga & folha & Sumo & Dor de cabeça \\
\hline
\end{tabular}

Fonte: Autor (2019). 
Evidencia-se que as famílias identificadas apresentam ampla distribuição e também frequentemente usadas na cura de várias doenças por comunidades amazônicas, conforme observado por Rodrigues (2006), Coelho-Ferreira (2009), Judd et al. (2009) e Vásquez et al. (2014).

Outra variável analisada diz respeito ao hábito vegetativo das espécies, onde a maioria é classificada como erva (35), seguidas de subarbusto (20) e arbusto (8) (Figura 2).

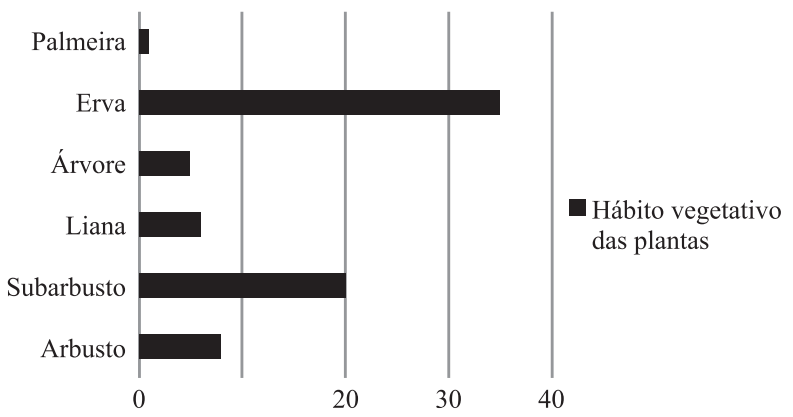

Figura 2 - Hábito vegetativo das espécies identificadas na comunidade quilombola Conceição de Mirindeua (Moju-PA).

Fonte: Autor (2019).

Esses resultados são similares aos encontrados no estudo de Ferreira (2000), sobre o levantamento de plantas medicinais em Marudá-PA, no qual a maioria era composta de espécies herbáceas, cerca de 94 (41,22\%), seguidas de arbóreas, 65 (28,50\%) e arbustivas 35 (15,35\%). De acordo com Guarim-Neto \& Amaral (2010), as ervas se encontram em maior quantidade de cultivo por se tratarem de plantas que ocupam pouco espaço nas propriedades.

Intentou-se também apresentar dados a respeito da origem dessas espécies, ou seja, demonstrar se essas são nativas ou oriundas de outros países do mundo, os dados foram tabulados através de informações obtidas de referências bibliográficas. A Tabela 2 exemplifica os resultados encontrados.

Tabela 2 - Origem das espécies identificadas

\begin{tabular}{lcc}
\hline Origem & Número absoluto & $\%$ \\
\hline Nativas & 31 & 37,34 \\
Exóticas & 52 & 62,65 \\
\hline Total & 83 & 100 \\
\hline
\end{tabular}

Fonte: Autor (2009).
Os resultados obtidos através dos questionários aplicados demonstraram que as espécies mais citadas e/ ou cultivadas pelos diversos detentores dos conhecimentos sobre plantas medicinais foram: Cipó Alho Mansoa alliaceae (Lam.) A.H. Gentry; Canarana Costus spicatus (Jacq.) Sw.; Mucuracá Petiveria alliacea L.; Arruda Ruta graveolens L.; Pirarucu Kalanchoe crenata (Andrews) Haw.; Algodão Gossypium arboreum L.; Catinga de mulata Aeollanthus suaveolens Mart. ex Spreng.; Hortelãzinho Mentha pulegium L.; Mastruz Dysphania ambrosoides (L) Mosyakin \& clemants.; Boldo Gymnanthemum amygdalinum (Delile) Sch.Bip. Ex Walp. (Figura 3).

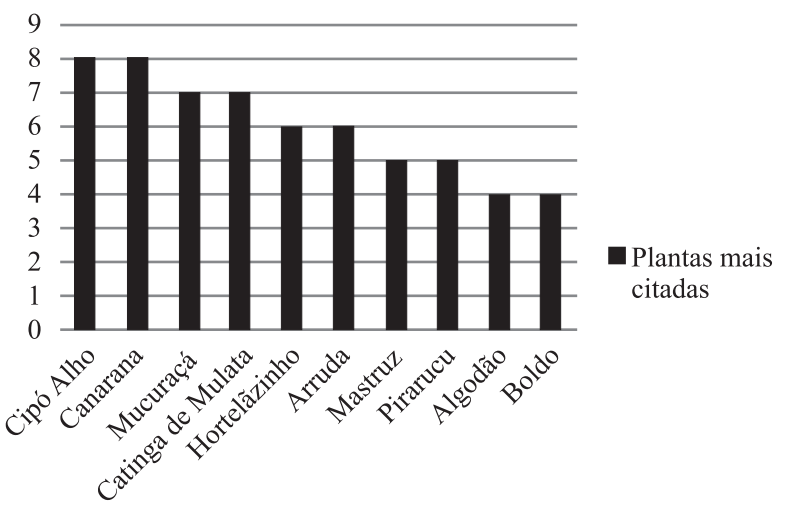

Figura 3 - Plantas mais citadas na comunidade quilombola Conceição de Mirindeua (Moju-PA).

Fonte: Autor (2019).

As espécies encontradas no levantamento etnobotânico também possuem grande importância em diversas comunidades tradicionais quilombolas como no Quilombo Sangrador (MA) (Monteles \& Pinheiro, 2007).

Constatou-se no trabalho que todas as partes das plantas são utilizadas no preparo dos fitoterápicos, porém a mais citada foi a folha $(73,25 \%)$, seguida da raiz $(9,30 \%)$ e casca $(6,97 \%)$. (Figura 4).

Pesquisas de plantas medicinais comumente demostram que a folha é a parte vegetal mais utilizada para fins de remédios caseiros (Coelho-Ferreira \& Jardim, 2005; Leão et al., 2007; Aguiar \& Barros, 2012).

Além disso, é a parte que mais interage diretamente com o ambiente, apresentando inúmeras especificidades para o tratamento de doenças (Rempel et al., 2019). Sua grande vantagem é que pode ser cultivada em uma grande variedade de solos e climas e sempre está disponível em maior parte do tempo, diferente de outras estruturas já que 
sementes, flores e frutos não se encontram disponíveis em outras épocas do ano (Cajaiba et al., 2016).

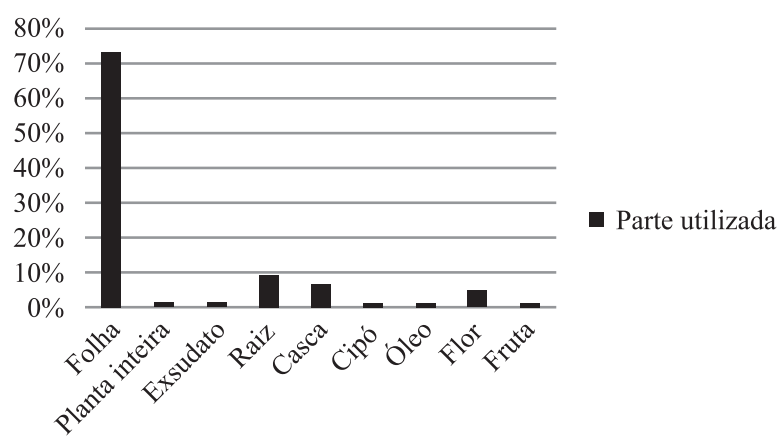

Figura 4 - Partes das plantas utilizadas para uso medicinal na comunidade quilombola Conceição de Mirindeua (Moju-PA).

Fonte: Autor (2019).

A forma de preparo mais comum das receitas caseiras é o chá $(61,62 \%)$, seguido pelo banho $(12,79 \%)$ e sumo $(10,46 \%)$ (Figura 5).

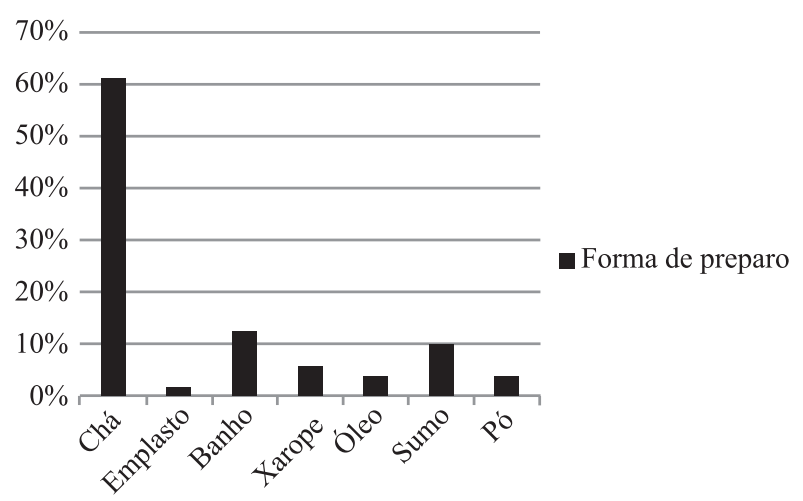

Figura 5 - Formas de preparo mais citadas de acordo com o levantamento na comunidade quilombola Conceição de Mirindeua (Moju-PA).

Fonte: Autor (2019).

O chá destaca-se, pois pode ser obtido através da infusão, da decocção (cozimento) e da maceração. Também apresenta habilidade de gerar inúmeros benefícios à saúde, o que mobiliza o interesse por pesquisas sobre sua ingestão. Além disso, a utilização frequente da bebida pode acarretar benefícios ao tratamento de inúmeras patologias (Moraes \& Souza, 2016).
As doenças mais tratadas através do uso de fitoterápicos são as do aparelho digestivo (18\%), do aparelho respiratório (10\%), do sistema cardiovascular $(6 \%)$ e também as doenças do sistema tegumentar (4\%) (Figura 6).

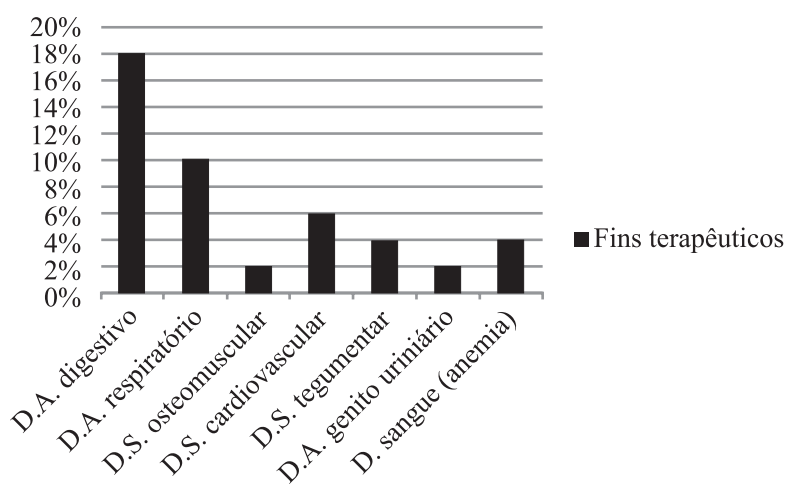

Figura 6 - Fins terapêuticos mais citados na comunidade quilombola Conceição do Mirindeua (MojuPará).

Fonte: Autor (2019).

Os resultados obtidos corroboram os encontrados por Pinto, Amorozo \& Furlan (2006) num estudo semelhante em comunidades rurais de mata atlântica em Itacaré-BA, no qual doenças relacionadas ao sistema digestivo e ao respiratório estão entre as mais citadas, com $21,4 \%$ e $10,8 \%$ do total de citações, respectivamente.

Além das doenças citadas com utilização de fitoterápicos, foram citadas plantas para uso de tratamentos espirituais com quatro citações.

Dentre os entrevistados, $76 \%$ afirmam ter acesso a serviços de saúde e $24 \%$ dizem não ter acesso, visto que a comunidade não possui unidade básica de saúde. Dessa forma, é notória a importância que as plantas medicinais desempenham na prevenção e tratamento de doenças dentro das comunidades tradicionais do município, onde geralmente, o sistema de saúde inexiste ou funciona de maneira precária.

As plantas produzem uma grande variedade de substâncias químicas que podem apresentar diversas atividades biológicas e constituem ainda hoje um recurso terapêutico relevante para uma parcela significativa da população mundial que não tem acesso aos medicamentos industrializados (Tôrres et al., 2005).

Todos os entrevistados afirmaram nunca terem sentido quaisquer efeitos colaterais advindos da utilização 
de fitoterápicos, pois os mesmos acreditam não haver efeitos adversos, como de toxicidade, por se tratar de produtos naturais. No entanto, existem evidências que algumas espécies podem sim apresentar riscos à saúde se não tomadas as devidas precauções. O maracujá (Passiflora edulis Sims), por exemplo, possui algumas substâncias químicas que podem causar efeitos tóxicos a saúde. Por conta disso, é recomendável a fervura demorada do chá para eliminá-las, além de evitar doses altas e tratamentos por longos períodos (Matos, 2000; Lorenzi \& Matos, 2008).

Outro exemplo é a planta quebra-pedra (Phyllanthus niruri L.) que não deve ser utilizada além das doses recomendadas pois pode provocar ação tóxica dos alcaloides presentes e também não deve ser usada durante a gestação e lactação, pois suas propriedades atravessam a placenta e podem ser excretadas pelo leite materno, além de ser abortivo e purgativo (Martins, 1994; Sartório et al., 2000; Rio de Janeiro - Estado, 2002; Torres, 2005; Lorenzi \& Matos, 2008).

Assim, fica evidente a necessidade de propostas de intervenção voltadas a repassar informações a respeito da toxicidade de algumas plantas e a importância do uso racional destas.

\section{CONCLUSÃO}

$\mathrm{Na}$ comunidade quilombola Conceição do Mirindeua foram levantadas 86 espécies vegetais utilizadas para fins medicinais onde as enfermidades mais tratadas foram as doenças do aparelho digestivo e respiratório. A maioria das espécies foram cultivadas nos quintais com destaque para a família Lamiaceae. Em relação ao preparo dos remédios caseiros a forma de chá foi a mais citada sendo as folhas a parte mais utilizada.

Evidencia-se que o uso de plantas medicinais é frequente na comunidade e muito valorizado por uma significativa parcela dos moradores, principalmente os mais antigos que, geralmente, são de baixa renda e analfabetos ou com baixo nível de escolaridade. Para estes, a utilização de plantas medicinais configura-se como a mais viável ou até mesmo única alternativa diante dos inúmeros problemas e dificuldades relacionados ao acesso a serviços básicos de saúde da localidade.

Diante disso, ressalta-se a importância de novas pesquisas e aprofundamento das já existentes sobre a utilização de plantas medicinais pelas populações tradicionais da Amazônia, uma vez que este é um assunto de interesse científico quanto social.

\section{LITERATURA CITADA}

AGUIAR, L.C.G.G.; BARROS, R.F.M. Plantas medicinais cultivadas em quintais de comunidades rurais no domínio do cerrado piauiense (Município de Demerval Lobão, Piauí, Brasil). Revista Brasileira de Plantas Medicinais, v.14, n.3, p.419-434, 2005.

ALMEIDA, V.S.; BANDEIRA, F.P.S.F. O significado cultural do uso de plantas da Caatinga pelos quilombolas do Raso da Catarina, municípió de Jeremoabo, Bahia, Brasil. Rodriguésia, v.2, n.61, p.195-209, 2010.

BAILEY, K. Methods of social research. 4.ed. New York: The Free Press, 1994. 588p.

BORBA, A.M.; MACEDO, M. Plantas medicinais usadas para a saúde bucal pela comunidade do bairro Santa Cruz, Chapada dos Guimarães, MT, Brasil. Acta Botanica Brasilica, v.20, n.4, p.771-782, 2006.

BRUNING, M.C.R.; MOSEGUI, G.B.G.; VIANNA, C.M. de M. A utilização da fitoterapia e de plantas medicinais em unidades básicas de saúde nos municípios de Cascavel e Foz do Iguaçu - Paraná: a visão dos profissionais de saúde. Ciênc. Saúde coletiva, Rio de Janeiro, v.17, n.10, p.2675-2685, 2012.

CAJAIBA, R.L.; da SILVA, W.B.; de SOUSA, R.D.N.; de SOUSA, A.S. Levantamento etnobotânico de plantas medicinais comercializadas no município de Uruará, Pará, Brasil. Biotemas, v.29, n.1, p.115-131, 2016.

CASSAS, F.; SILVA, D.S.; BARROS, C.; REIS, N.F.C.; RODRIGUES, E. Canteiros de plantas medicinais, condimentares e tóxicas como ferramenta de promoção à saúde no jardim botânico de Diadema, SP, Brasil. Revista Ciência Ext., v.12, n.2, p.37-46, 2016.

COELHO-FERREIRA, M. Medicinal knowledge and plant utilization in an Amazonian coastal community of Marudá, Pará State (Brazil). Journal of Ethnopharmacology, v.126, n.1, p.159-175, 2009.

COELHO-FERREIRA, M.R.; JARDIM, M.A.G. Algumas espécies vegetais usadas pelos moradores da ilha de Algodoal, Maiandeua, município de Maracanã, Pará. Boletim do Museu Paraense Emílio Goeldi, v.1, n.2, p.4551, 2005.

DIEGUES, A.C. Etnoconservação: novos rumos para a conservação da natureza. Annablume, São Paulo, 2000. 290 p.

FERREIRA, M.C. Identificação e valorização das plantas de uma comunidade pesqueira do litoral paraense. Dissertação (Mestrado-Área de Concentração em 
Botânica). Belém, PA: Universidade Federal do Pará, 2000. 269p.

GIRALDI, M.; HANAZAKI, N. Uso e conhecimento tradicional de plantas medicinais no Sertão do Ribeirão, Florianópolis, SC, Brasil. Acta Bot. Bras., v.24, n.2, p.395406, 2010.

GUARIM NETO, G.; AMARAL, C.N. Aspectos etnobotânicos de quintais tradicionais dos moradores de Rosário Oeste, Mato Grosso, Brasil. Polibotanica, n.29. p.191-212, 2010.

IBGE, Dados sociodemográficos do município de Moju. 2010 in: https://cidades.ibge.gov.br/brasil/pa/moju/ panorama. (Acessado em 28 de novembro de 2019).

ITERPA Instituto de Terras do Pará. In: http://www.iterpa. pa.gov.br/quilombolas. (Acessado em: 27 de novembro de 2019).

JUDD, W.S.; CAMPBELL, C.S.; KELLOGG, E.A.; STEVENS, P.F.; DONOGHUE, M.J. Sistemática Vegetal: um enfoque filogenético. 3.ed. Porto Alegre: Artmed, 2009. $612 \mathrm{p}$.

LEÃO, R.B.A.; COELHO-FERREIRA M.R.; JARDIM, M.A.G. Levantamento de plantas de uso terapêutico no município de Santa Bárbara do Pará, Estado do Pará, Brasil. Revista Brasileira de Farmácia, v.88, p.21-25, 2007.

LORENZI, H.; MATOS, F.J.A. Plantas medicinais no Brasil: nativas e exóticas. 2.ed. Nova Odessa: Instituto Plantarum, 2008.

MARTINS, F.R. O método de quadrantes e a fitossociologia de uma floresta residual no interior do Estado de São Paulo: Parque Estadual de Vassununga. Tese (Doutorado- Área de Concentração em Ciências). São Paulo-SP: Universidade de São Paulo, 1979. 239p.

MATOS, F.J.A. Plantas medicinais - Guia de seleção e emprego de plantas usadas em fitoterapia no Nordeste do Brasil. 2.ed. Fortaleza: Imprensa Universitária da UFC, 2000.

MONTELES, R.; PINHEIRO, C.U.B. Plantas medicinais em um quilombo maranhense: uma perspectiva etnobotânica. Revista de Biologia e Ciências da Terra, v.7, n.2, p.1-12, 2007.

MORAES, A. da S.M.; SOUZA, V.R.S. Chá Verde e Suas Propriedades Funcionais nas Doenças Crônicas não Transmissíveis. Revista Interdisciplinar Pensamento Cientifico, v.2, n.1, 2016.

NASCIMENTO, J.M.; CONCEIÇÃO, G.M. Plantas medicinais e indicações terapêuticas da comunidade quilombola Olho d'água do Raposo, Caxias, Maranhão, Brasil. BioFar: Revista Brasileira de Biologia e Farmácia, v.6, n.2, p.138-151, 2011.

NODA, S.; NODA, H.; MARTINS, A.L.U. Papel do processo produtivo tradicional na conservação dos recursos genéticos vegetais. In: RIVAS A.; FREITAS, C.E.C. (Org.). Amazônia uma perspectiva interdisciplinar. Manaus: Editora da Universidade do Amazonas, 2002. p.155-178.

OLIVEIRA, C.J; ARAÚJO, T.L. Plantas medicinais: usos e crenças de idosos portadores de hipertensão arterial. Revista eletrônica de enfermagem, v.9, n.1 p.93-105, 2007.

PIMENTA, L.; BELTRÃO, N.; GEMAQUE, A.; PONTES, A. Dinâmica do uso e cobertura da terra em municípios prioritários: uma análise no município de Moju, Pará no período de 2008 a 2014. Revista de Geografia e Ordenamento do Território, n.14, p.267-281, 2018.

PINTO, E. de P.P; AMOROZO, M.C.M; FURLAN, A. Conhecimento popular sobre plantas medicinais em comunidades rurais de mata atlântica - Itacaré, BA, Brasil. Acta Bot. Bras., São Paulo, v.20, n.4, p.751-762, 2006.

REMPEL, C.; MACIEL, M.J.; BERGMANN, P.C.; de BORBA M.A.P.; GOETTENS, C. Efeito antimicrobiano de plantas medicinais: uma revisão de estudos científicos. Revista Ibero-Americana de Ciências Ambientais, v.10, n.4, p.57-82, 2019.

RIGONATO, V.D.; ALMEIDA, M.G. As fitofisionomias e a interrelação das populações tradicionais com o bioma cerrado. In: Cultura, conhecimento popular e uso das espécies nativas pelos pequenos agricultores do Cerrado. POCPG/CNPq. 2003.

RIO DE JANEIRO, Estado. Secretaria de Estado de Saúde. Resolução SES/RJ no 1757, 18 de fevereiro de 2002. Contraindicações e o uso de plantas medicinais no Âmbito do Estado do Rio de janeiro e dá outras providências. Diário Oficial do Estado, Rio de Janeiro, 20 fev. 2002. Ano XXII, n. 33. Parte I. Revista Episteme Transversalis, v.3, n.1, 2012. CENTRO UNIVERSITÁRIO GERALDO DI BIASE.

RODRIGUES, E. Plants and animals utilized as medicines in the Jaú National Park (JNP). Brazilian Amazon Phytotherapy, v.20, n.5, p.378-391, 2006.

SÁ, M.L.B. Saberes e práticas alimentares em uma comunidade quilombola no Ceará. 2010. Tese (Doutorado em Saúde Pública). São Paulo-SP: Faculdade de Saúde Pública da Universidade de São Paulo, 2010.

SALES, M.D.C.; SARTOR, E.B.; GENTILLI, R.M.L. Etnobotânica e Etnofarmacologia: medicina tradicional 
e bioprospecção de fitoterápicos. Revista Salus, v.1, n.1, p.17-26, 2015.

SANTOS, R.C.; SILVA, M.S. Condições de vida e itinerários terapêuticos de quilombolas de Goiás. Fonte de financiamento: Conselho Nacional de Desenvolvimento Científico e Tecnológico (CNPq). Saúde e Sociedade [online]. v.23, n.3, p.1049-1063, 2014.

SARTÓRIO, M.L. et al. Cultivo orgânico de plantas medicinais. Viçosa, MG: Aprenda fácil. 2000.

SEPPIR - SECRETARIA DE POLÍTICAS DE PROMOÇÃO DA IGUALDADE RACIAL. Programa Brasil Quilombola: diagnóstico das ações realizadas. Brasília, DF, 2012.

SILVA, N.C.B.; REGIS, A.C.; ESQUIBEL, M.A.; SANTOS, J.E.S.; ALMEIDA, M.Z. Uso de plantas medicinais na comunidade quilombola da Barra II - Bahia, Brasil. Boletín Latino Americano y Del Caribe de Plantas Medicinales y Aromáticas, v.11, n.5, p.435-453, 2012.
SIVIERO, A. et al. Plantas medicinais nos quintais urbanos de Rio Branco, Acre. Rev. bras. Plantas med., Botucatu, v.14, n.4, p.598-610, 2012.

TÔRRES, A.R. et al. Estudo sobre o uso de plantas medicinais em crianças hospitalizadas da cidade de João Pessoa: riscos e benefícios. Revista Brasileira de Farmacognosia, v.15, n.4, p.373-380, 2005.

TORRES, P.G.V. Plantas medicinais, aromáticas \& condimentares: uma abordagem prática para o dia-a-dia. Porto Alegre: Editora Rígel. 2005.

VÁSQUEZ, S.P.F.; MENDONÇA, M.S.; NODA, S.N. Etnobotânica de plantas medicinais em comunidades ribeirinhas do Município de Manacapuru, Amazonas, Brasil. Acta Amazônica. v.44, n.4, p.457-472, 2014.

WINKLERPRINS, A.; OLIVEIRA, P.S. Urbanagriculture in Santarém, Pará, Brazil: diversity and circulation of cultivated plants in urban homegardens. Boletim do Museu Paraense Emílio Goeldi, v.5, n.3, p.571-585, 2010.

Recebido para publicação em 07/02/2020, aprovado em 04/04/2020 e publicado em 30/04/2020. 